\title{
Bearing Fault Detection Based on TQWT and Hilbert Envelope
}

\author{
Qi Liu
}

School of Economics and Management, Jiangsu University of Science and Technology, Zhenjiang 212000, China.

qiliu_JUST@126.com

Keywords: Bearing, fault detection, TQWT, Hilbert envelope.

\begin{abstract}
Bearings always work under harsh environment, which makes them easily to failure. Fault detection of the bearing is a key issue for maintaining the whole machinery system. In this paper, a novel method is proposed to detect the fault of bearings. Tunable Q-factor wavelet transform (TQWT) is used to decompose the original vibration bearing signal to several levels, the Hilbert envelope analysis method is applied to each wavelet decomposed signal. The fault feature of the bearing can be detected from the envelope spectrum. Both the simulation study and the application demonstrate the effectiveness of the proposed method.
\end{abstract}

\section{Introduction}

Detection of transients has always been an important task in signal processing, and it has also been increasingly significant in the field of machinery fault diagnosis in recent years [1]. The fault of bearings may lead to the failure of the whole machinery system. Thus, proper analysis for fault detection from machinery vibration signals has attracted sustained attention during the past decades [2].

Vibration signals measured from the fault bearings are generally observed as periodic transient impulses on the constant speed. Recently, many different methods have derived for fault detection, such as the Fourier transform, short-time Fourier transform, wavelet transform, etc. Wavelet transform uses a series of windows to process the signal, thus can provide the information within a certain time. A new wavelet transform called tunable Q-factor wavelet transform (TQWT) is proposed by Selesnick [3] in recent years. Application of TQWT in machinery fault detection has proven its effectiveness [4]. However, when the background noise is strong, TQWT cannot precisely extract the fault features.

Hilbert envelope spectrum [5] is another classical method which has been widely used in fault detection of machinery. By incorporating the TQWT and Hilbert envelope spectrum, a novel method is proposed in this paper. Firstly, TQWT is applied to decompose the original vibration signal to several levels, then Hilbert envelope analysis method is used to analyze each wavelet decomposed signal. The fault feature of the bearing can be found in the envelope spectrum.

The remaining parts are organized as follows. Firstly, the theoretical basis of the method is introduced, which includes the TQWT and Hilbert envelope analysis method. Then simulations with different signal to noise ratios (SNR) are conducted to verify the proposed method. Then the proposed early fault detection method is applied to bearing fault feature extraction. Finally, conclusions are drawn in the last section.

\section{Problem Formulation}

Tunable Q-factor Wavelet Transform. The Q factor, which is used as a quantification of the oscillatory nature of a signal transient, affects the oscillatory behavior of the wavelet basis function. TQWT, whose Q factor can be tuned, is proposed to analyze the discrete time signal. There are two important factors in TQWT, one is the Q factor, another is the redundancy. Q measures the number of oscillations in the wavelet basis function, higher $\mathrm{Q}$ factor means more oscillatory cycles. Redundancy $r$ is used to measure the total over-sampling rate of TQWT. Suppose the TQWT decompose the original signal into $\mathrm{J}$ levels, the relations between the parameters are 


$$
\mathrm{Q}=\frac{2}{\beta}-1, \mathrm{r}=\frac{\beta}{1-\alpha}, \mathrm{J}=\frac{\log (\beta \mathrm{N} / 8)}{\log (1 / \alpha)}
$$

where $\alpha$ is the low-pass scaling parameter, $\beta$ is the high-pass scaling parameter, $\mathrm{N}$ is the length of the signal. Fig. 1 shows that TQWT iteratively applies two-channel filter banks on the low-pass channel.

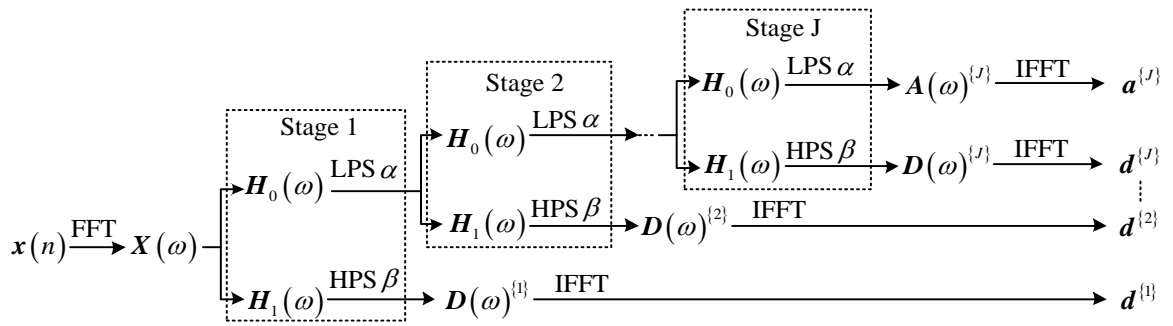

Fig. 1. The procedure of the tunable Q factor wavelet transform.

After TQWT, the original signal $\mathrm{x}(\mathrm{t})$ will be decomposed to the scaling coefficientsa $\mathrm{JU}^{\mathrm{J}\}}$ and $\mathrm{J}$ wavelet coefficients d

$$
\mathbf{d}=\left\{\mathbf{d}^{\{1\}}, \mathbf{d}^{\{2\}}, \ldots, \mathbf{d}^{\{J\}}\right\}
$$

Hilbert Envelope. Given a real time signal $\mathrm{x}(\mathrm{t})$, the Hilbert transform $\mathrm{h}(\mathrm{t})=\mathrm{H}\{\mathrm{x}(\mathrm{t})\}$ is defined as

$$
\mathrm{h}(\mathrm{t})=\mathrm{H}\{\mathrm{x}(\mathrm{t})\}=\frac{1}{\pi} \int_{-\infty}^{\infty} \frac{\mathrm{x}(\tau)}{\mathrm{t}-\tau} \mathrm{d} \tau=\frac{1}{\pi \mathrm{t}} * \mathrm{x}(\mathrm{t})
$$

thus, $h(t)$ is obtained as the convolution of the function $\frac{1}{\pi t}$ and the original signal. The real signal $\mathrm{x}(\mathrm{t})$ and its Hilbert transform $\mathrm{h}(\mathrm{t})$ can form a new complex signal, which is called the analytical signal, defined as

$$
\mathrm{z}(\mathrm{t})=\mathrm{x}(\mathrm{t})+\mathrm{jh}(\mathrm{t})
$$

The envelope $\mathrm{E}(\mathrm{t})$ of the complex signal $\mathrm{z}(\mathrm{t})$ is defined as

$$
\mathrm{E}(\mathrm{t})=|\mathrm{z}(\mathrm{t})|=\sqrt{\mathrm{x}^{2}(\mathrm{t})+\mathrm{h}^{2}(\mathrm{t})}
$$

By performing the spectrum analysis to the envelope signal $E(t)$, the envelope spectrum can be got.

When the fault signal is decomposed by the TQWT into several levels, the Hilbert envelope analysis method is applied to the decomposed signals. The fault frequencies will appear in the envelope spectrum, which can be used a reliable source of information for bearing fault diagnosis.

\section{Simulation Study}

A simulation case is performed to verify the effectiveness of the proposed method. Considering the characteristics of the vibration signal of a faulty bearing, a periodic signal $x(t)$ is constructed as:

$$
x(t)=A_{s} \sum_{k} e^{\frac{-\zeta_{0}}{\sqrt{1-\zeta_{0}^{2}}} 2 \pi f_{0}\left(t-k T_{0}-\tau_{0}\right)} \sin \left(2 \pi f_{0}\left(t-k T_{0}-\tau_{0}\right)\right)+n(t)
$$

where frequency $f_{0}=200 \mathrm{~Hz}$, damping ratio $\zeta_{0}=0.05$, time index $\tau_{0}=0.1 \mathrm{~s}$, cyclic period $\mathrm{T}=0.2 \mathrm{~s}$ and the noise $\mathrm{n}(\mathrm{t})$ is Gaussian white noise $\mathrm{n}(\mathrm{t}) \sim \mathrm{N}\left(\mathbf{0}, \boldsymbol{\sigma}^{2}\right), \boldsymbol{\sigma}^{2}$ can be estimated from the normal condition. Here we set $\boldsymbol{\sigma}^{2}=\mathbf{I}$. The original signal and the noisy signal are shown in Fig.2, where SNR is used to weigh the noise level which is defined as

$$
\mathrm{SNR}=10 \times \lg \left(\frac{\mathrm{P}_{\mathrm{s}}}{\mathrm{P}_{\mathrm{n}}}\right)
$$

where $P_{s}$ is the energy of the faulty signal and $P_{n}$ is the energy of the noise. 

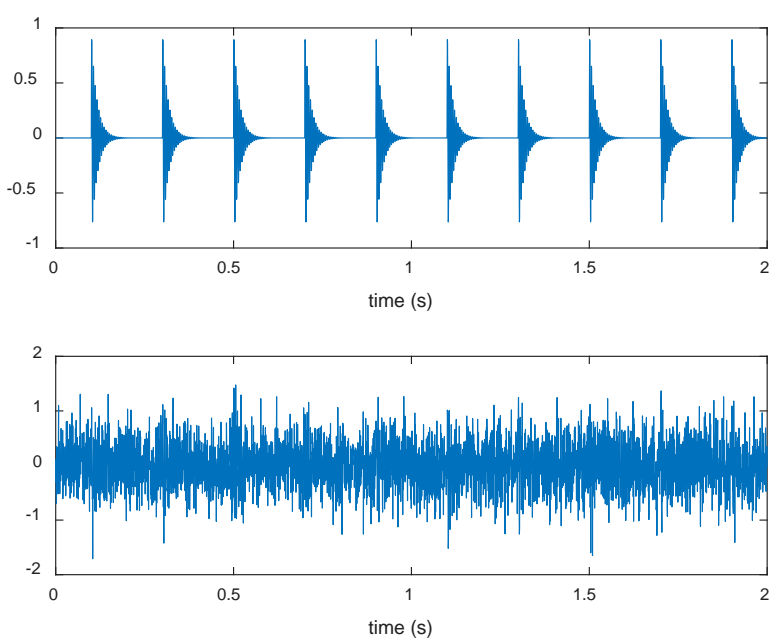

Fig. 2 The original and noisy (SNR=-8.873dB) simulation signal.

Apply the proposed method to the noisy vibration signal. First, decompose the signal into J levels with $\mathrm{Q}=6, \mathrm{r}=3, \mathrm{~J}=6$. The decomposed signals are shown in Fig.3 (a). Then the Hilbert envelope spectrums of the decomposed signals are illustrated in Fig.3 (b). From the sixth figure in Fig.3 (b), we can obtain the fault frequency as $5 \mathrm{~Hz}$, which is the fault feature of the simulated signal.
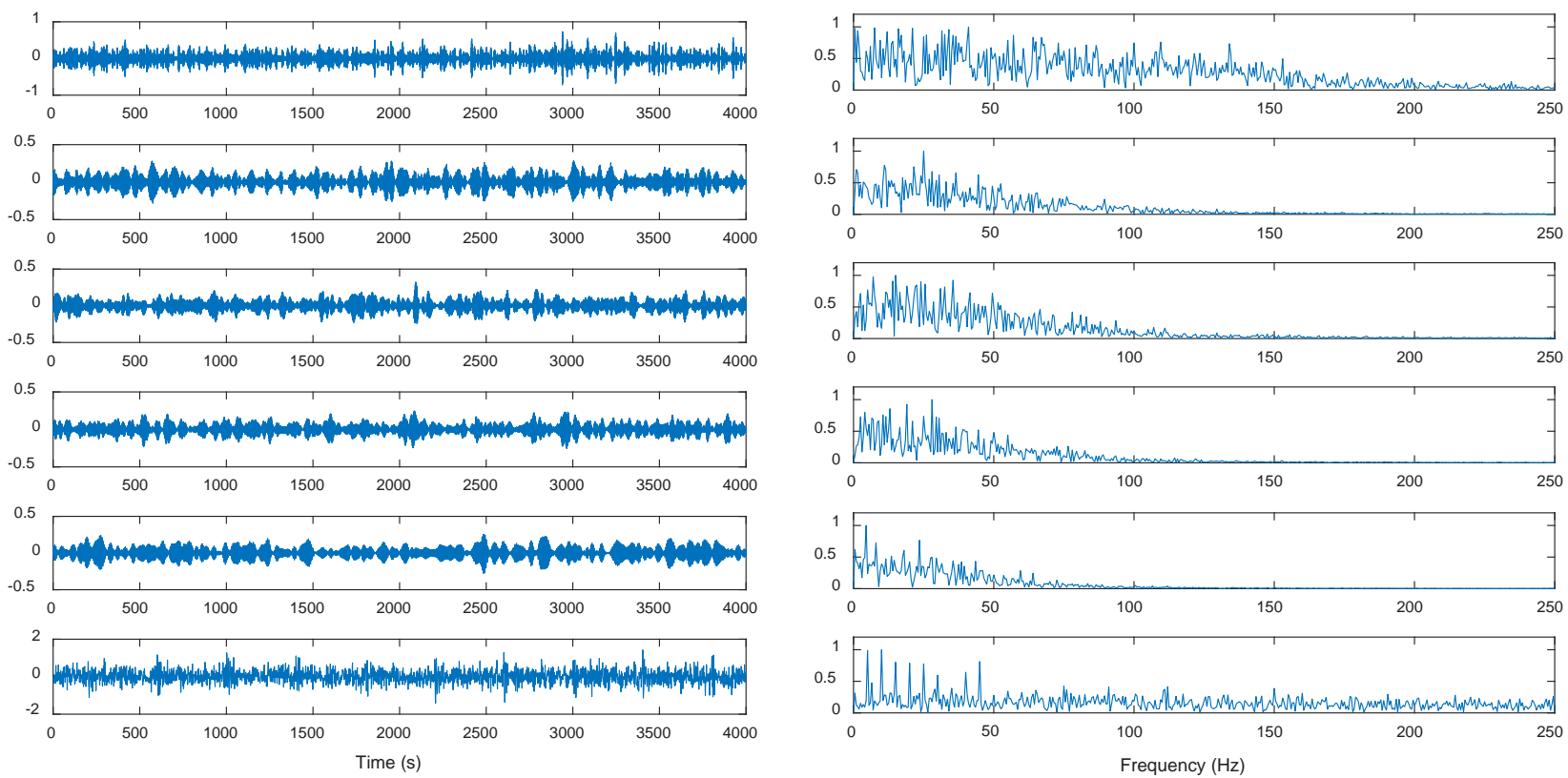

(a)

(b)

Fig. 3 (a) The decomposed signals by TQWT; and (b) the envelope spectrum of the decomposed signals.

\section{Application to Fault Detection}

The bearing data from CWRU are used to demonstrate the effectiveness of the proposed method. The vibration signal of outer race fault is displayed in Fig.4.

The proposed method is applied to analyze the vibration signal. First, decompose the signal into $\mathrm{J}$ levels with $\mathrm{Q}=6, \mathrm{r}=3, \mathrm{~J}=6$. The decomposed signals are shown in Fig.5 (a). Then the Hilbert envelope spectrums of the decomposed signals are illustrated in Fig.5 (b). From the sixth figure in Fig.5 (b), we can obtain the fault frequency as $107 \mathrm{~Hz}$, which is the fault feature of the simulated signal. 


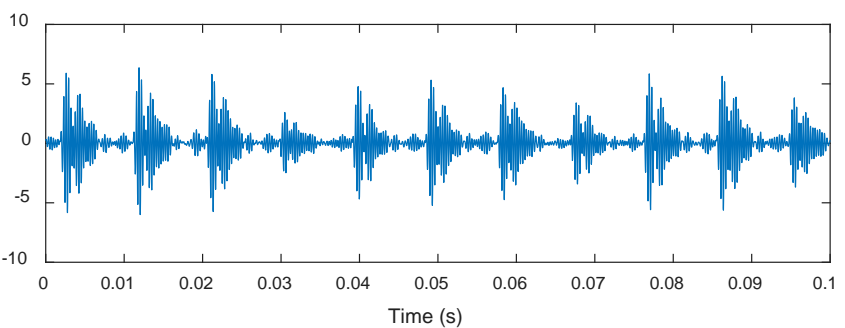

Fig. 4 The vibration signal of outer race fault.
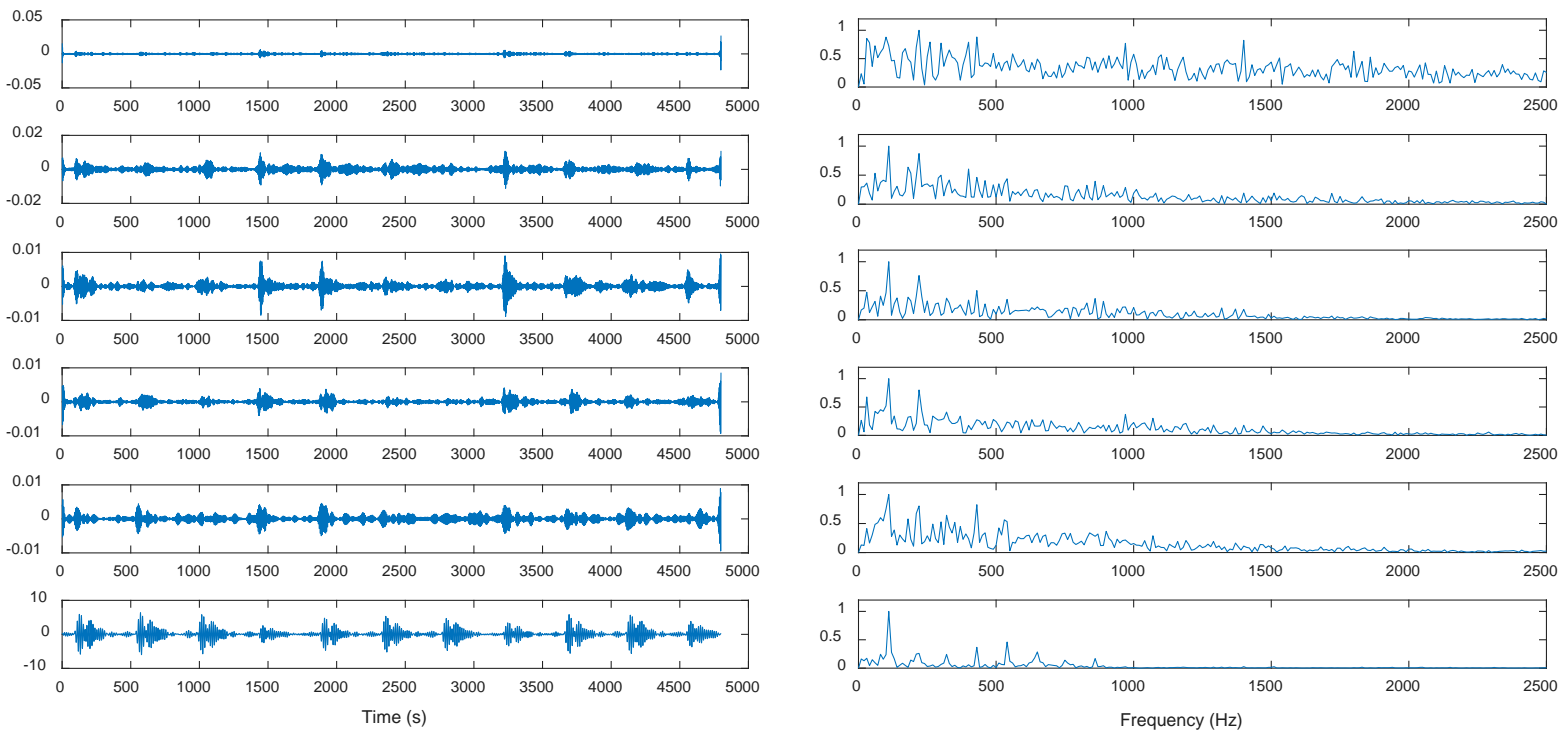

Fig. 5 (a) The decomposed signals by TQWT; and (b) the envelope spectrum of the decomposed signals.

\section{Summary}

A novel fault detection method based on TQWT and Hilbert envelope is proposed in this paper. The signal is decomposed into several levels by TQWT, then the Hilbert envelope is applied to analyze the decomposed signals. Both the simulation study and the application study show the effectiveness of the proposed method.

\section{References}

[1] Zhang Q, Zhao G, Shu L, et al, Research of dimensionless index for fault diagnosis positioning based on EMD, Journal of Computers, 27.1 (2016) 62-73.

[2] Fan W, Cai G, Zhu Z K, et al, Sparse representation of transients in wavelet basis and its application in gearbox fault feature extraction, Mechanical Systems and Signal Processing, 56 (2015) 230-245.

[3] Selesnick I W, Wavelet transform with tunable Q-factor, IEEE transactions on signal processing, 59.8 (2011) 3560-3575.

[4] Cai G, Chen X, He Z, Sparsity-enabled signal decomposition using tunable Q-factor wavelet transform for fault feature extraction of gearbox, Mechanical Systems and Signal Processing, 41.1 (2013) 34-53.

[5] Yang Y, Yu D, Cheng J, A fault diagnosis approach for roller bearing based on IMF envelope spectrum and SVM, Measurement, 40.9 (2007) 943-950. 